\title{
Philips Medical Systems Support for Open ECG and Standardization Efforts
}

\author{
ED Helfenbein, R Gregg, S Zhou \\ Advanced Algorithm Research Center \\ Diagnostic ECG Division, Philips Medical Systems, Milpitas, CA, USA
}

\begin{abstract}
Philips Medical Systems developed a new ECG data format based on XML technology in response to increased demands for ECG data access and better ECG device interoperability. Philips has published the schema for the XML-based ECG format that is used by its electrocardiographs, bedside monitors, and defibrillators. To assist access to ECG data, Philips provides users with a set of software tools for XML-ECG waveform manipulation and display.

In addition, Philips Medical Systems has been strongly supporting all national and international initiatives that are underway to standardize ECG formats and improve interoperability between diagnostic ECG devices and healthcare information systems.
\end{abstract}

\section{Introduction}

Demands for access to electrocardiographic (ECG) data and for better interoperability among ECG devices have increased drastically in recent years. In response to such increased demands, Philips Medical Systems developed a new ECG data format based on XML (Extensible Markup Language) technology and published the schema of the data format in 2003. An XML-based ECG format is extremely well-suited for computerized interchange of information, and XML resources and tools are readily available. All Philips electrocardiographs, bedside monitors and defibrillators share and export ECGs in this XML-based format. To further assist users' access to ECG data, Philips provides a set of software tools for XML-ECG waveform manipulation and display. This freely published set of converters can be used to transform the Philips XML ECGs into a number of formats, including Health Level 7 (HL7)/Food and Drug Administration (FDA) Annotated ECG, XML with plain text decompressed waveform values, and Scalable Vector Graphics (SVG) allowing ECG report viewing with an SVG enabled web browser. A PC-based ECG receiver program also supports electrocardiograph connectivity.

On both the national and international levels, a number of efforts have been initiated to improve interoperability between diagnostic ECG devices and healthcare information systems. The HL7 Annotated ECG format adopted by the U.S. FDA for drug clinical trial ECG digital submissions has strengthened the movement towards common and publicly accessible ECG formats. The recently formed ACC (American College of Cardiology) sponsored IHE (Integrating the Healthcare Enterprise) Cardiology Technical Committee has developed a technical framework for retrieving and displaying diagnostic quality ECGs throughout the healthcare environment. Finally, the European Commission sponsored OpenECG project has initiated extensive discussions in the past two years regarding open ECG formats. As a major manufacturer of electrocardiographs, patient monitors, defibrillators and ECG management systems, Philips Medical Systems has actively participated in these efforts.

\section{XML-based ECG File Format}

Until recently, the native format for ECG files produced by the major cardiograph manufacturers has been binary files with compression and encoding of waveform data. The compression and encoding schemes were not published, and easy access to the ECG waveform data itself by researchers and clinicians was not possible. In addition, the binary file format used to store patient demographics, measurement values, and automated interpretations forced users who wanted access to this information to write custom software based on descriptions of the binary file format. With the increased popularity and use of XML as a data interchange vehicle [1], it became clear that an XML file format for ECG storage and transmission would provide many advantages.

XML is "a W3C-endorsed standard for document markup. It defines a generic syntax used to mark up data with simple, human-readable tags"[2]. XML document files are essentially tree-like structures containing labeled or "tagged" elements. Both the tags and the data elements are strings of text. Because of this, readily available text editors and web browsers can be used to 
view the data in XML documents. There are also a variety of specialized XML editors, such as XMLSPY (Altova, Austria), and the <oXygen/> XML Editor (SyncRO Soft Ltd., Romania) designed specifically to work with XML documents. To gain programmatic access to data contained in XML documents, a variety of software libraries are available for $\mathrm{C}, \mathrm{C}++, \mathrm{C \#}$, Perl and java programming languages. Microsoft's MSXML library (msdn.microsoft.com/XML) is certainly one of the most commonly used libraries for this purpose.

XML itself is quite flexible, allowing use in a variety of domains, but XML documents must also conform to a strict grammar. "Well-formed" XML documents pass this grammar. XML also allows for the creation of "schemas", which describe both the allowable markup, (e.g., the location of the elements in the document tree), and the form of the data in each element. These schemas are written in one of many different XML schema languages. Two examples are the "document type definition" (DTD) defined by the XML 1.0 specification, and the World Wide Web Consortium (W3C) XML Schema Language (www.w3c.org). The DTD is quite limited in its ability to define constraints on the data contained in XML documents, whereas the XML Schema Language allows for precise specification of valid data elements. Most XML parser libraries and editors allow for the validation of a given XML document against its DTD or schema.

In March 2003, Philips Medical Systems released the PagewriterTouch cardiograph, which was the first cardiograph by a major manufacturer to use an XML file format for native ECG storage and transmission. At the same time, Philips provided the XML ECG schema for the ECG files, which was written in the W3C XML Schema Language. The schema was made available on the internet, and was also included with the electrocardiograph documentation. Distribution of this schema in the public domain allowed users to easily develop applications that include the patient demographics, and automated measurements and interpretations contained in the Philips' XML ECG files.

The ECG waveform data within the Philips XML ECG is compressed with a lossless algorithm, and encoded into characters using a base 64 encoding scheme. This string of characters is included in the XML file as the data in a single node. With this approach, both the clear benefits of XML for data interchange and the efficiency from data compression are employed.

Philips provides a suite of software tools to give users easy access to the compressed waveform data. These include object libraries that contain the decompression and base 64 decoding algorithms. Electrocardiograph users in different fields have used these software tools to read Philips ECG files. In addition, a decompression application in the form of a Windows PC based executable has been released, and freely distributed to electrocardiograph customers. This package will convert a Philips XML ECG file with compressed and encoded waveform data into a corresponding XML file with the waveform data represented in plain-text values.

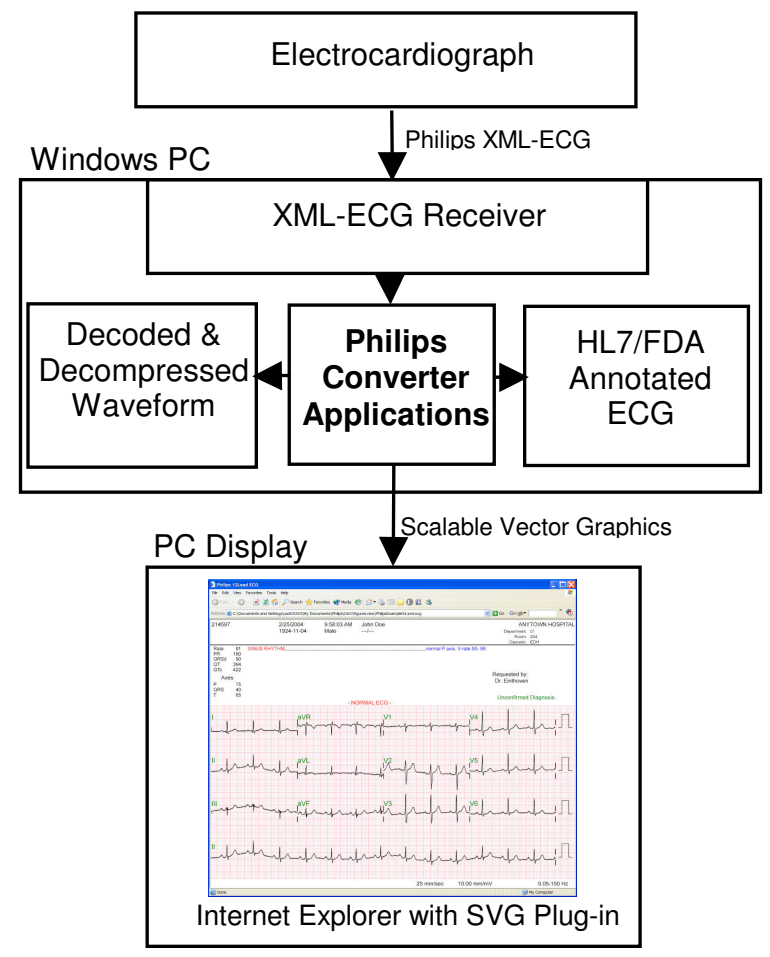

Fig.1: Illustration of software tools for XML-ECG manipulation and display. These allow connectivity between Philips cardiographs and other systems, open access to waveform data, standard formats, and presentation of ECG reports in a web browser

\section{Connectivity of ECG Devices}

All products with 12-lead ECG acquisition and storage capability across Philips Medical Systems product lines, including the PagewriterTouch \& PagewriterTrim electrocardiographs, Intellivue bedside monitor, HeartStartMRx defibrillator, TraceMasterVue ECG Management System [3], the IntelliVue Patient Information Center, and the Epic Hospital Information System, share the same XML ECG format. This use of a common format permits an increased efficiency of product interconnectivity development, testing, and support.

To provide connectivity to ECG devices, Philips distributed an application that allows a PC to receive ECGs directly from Philips electrocardiographs via a network. 


\section{SVG for ECG Display}

An important component of an open ECG environment is the ability to easily view ECG data in a graphical format. To address this user need, Philips turned to Scalable Vector Graphics (SVG), the XML-based graphics language, also standardized by the W3C.

SVG is a powerful and flexible XML application language, ideally suited for display of two-dimensional graphics on a variety of display devices [4]. Data in an XML document can be readily transformed into an SVG XML document. An SVG document can be rendered onto a display device simply by opening it with an SVG enabled web browser. Adobe's SVG plug-in [5] for Microsoft's Internet Explorer is one such example. SVG images are rendered when displayed, and rendered appropriately to the resolution of the display device, unlike the display of bitmapped images. The Adobe plug-in has a built-in capability that allows easy zooming and panning of the graphical data, ideally suited for display of high-resolution ECG waveforms. Thus, its use for display of Philips XML ECG data was a natural progression.

Philips created a software tool to convert Philips XML ECGs into SVG XML files, which could then be used to easily view Philips ECGs on any PC with a web browser with the SVG plug-in. This SVG converter has been distributed to users. A number of hospitals have employed this software package to display ECG reports within the hospitals' HIS system.

\section{Recent Efforts in ECG File Format Standardization}

\section{HL7/FDA Annotated ECG}

A few years ago, when it became apparent that noncardiac medications could have profound effects on cardiac repolarization, and hence susceptibility to sudden cardiac arrest [6], the U.S. FDA began requiring that all pharmaceutical clinical trials include diagnostic ECG collection and monitoring. Since they were about to be inundated with massive quantities of paper ECGs, the FDA turned to HL7 [7], an industry-wide consortium, to develop a standardized format for ECG files to be submitted in clinical trials. It was clear that since clinical trial ECGs are collected on cardiographs of varying vintage from numerous manufacturers, a plethora of proprietary format ECGs could not be systematically viewed for monitoring by the FDA.

The HL7 Regulated Clinical Research Information Management Technical Committee (RCRIM), with the assistance of representatives from Philips as well as other major manufacturers, developed the "Annotated ECG"
(aECG) file format. This standardized HL7 version 3 message (i.e. format) provides a flexible wrapper to contain ECG waveforms (including representative beat complexes) as well as both machine and manual measurements of intervals and amplitudes. The latest version of this format is from the Dec. 2003 ballot. A freeware aECG viewer is also provided from AMPS, LLC (www.amps-llc.com) and is available online.

The major electrocardiograph manufacturers have developed software applications on their ECG management systems for annotating ECGs with manual measurements (e.g., QT interval) and producing the aECG files. A demonstration of the entire process, from ECG acquisition, manual annotation, conversion to the aECG format, collection by a clinical trial sponsor, and submission to the FDA was performed at the Healthcare Information Management Systems Society (HIMSS)[8] conference in Feb. 2004 with participation by Philips as well as other organizations.

To further support this standardization effort, Philips also provides a PC based application for converting Philips XML ECGs into the aECG format.

\section{IHE Retrieve ECG for Display}

Integrating the Healthcare Enterprise (IHE) is "a multiyear initiative that creates the framework for passing vital health information seamlessly-from application to application, system to system, and setting to settingacross the entire healthcare enterprise" [10]. IHE was established in 1998 with support from HIMSS and the Radiological Society of North America, and drives the adoption of standards in different clinical areas. In March 2004, the Cardiology Technical Committee, with sponsorship from the ACC and participation by Philips and other cardiology diagnostic device companies, began work on a Technical Framework for three integration profiles. One of these profiles, "Retrieve Electrocardiograms for Display," provides a specification for a presentation format for ECG reports on a display device in a healthcare enterprise system [10].

The profile specifies a web application interface to an information source (such as an ECG management system) to retrieve an XML list of ECG reports available for a patient, as well as an XSLT style-sheet for presentation of the list in a web browser. Then, in response to a request for a particular ECG report, the profile provides a specification of the report presentation format in either PDF or SVG. Because PDF plug-in enabled web browsers (e.g., Adobe Acrobat Reader) are already ubiquitous in the healthcare environment, PDF was selected as a minimum required presentation format.

The IHE ECG standard also specifies the minimum information that must be present on the report, including 
patient demographics, calibration marks, and background grid. In addition, the profile addresses the need for high quality waveform presentations; it requires that PDF vector drawing content be created (e.g., from the Adobe PDF Library SDK) for the report generation, instead of creation of a bitmap image which may suffer from aliasing and image distortion when rendered on a display device.

IHE Retrieve ECG for Display

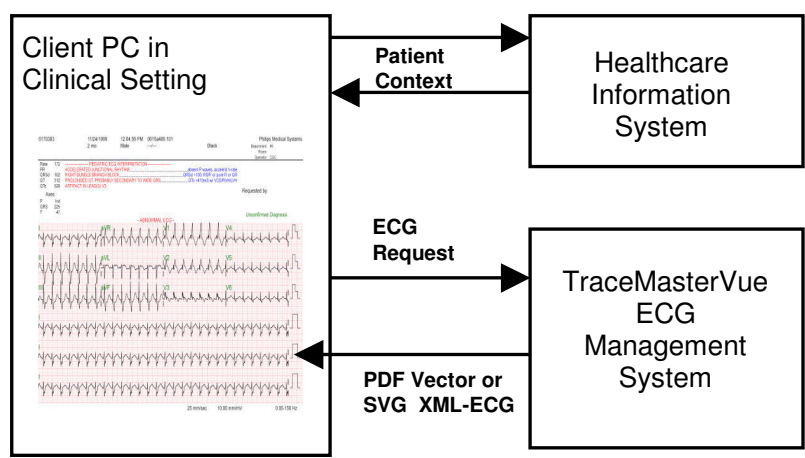

Fig.2: The IHE "Retrieve ECG for Display" specification provides a simple framework for presentation of ECG reports throughout the healthcare setting. Client PCs display ECG reports in a web browser with PDF or SVG plug-ins. SVG can permit client-side report format options via embedded scripting.

Recognizing the advantages of SVG for ECG presentation, the IHE profile also allows for the request of an SVG-based report instead of PDF. One particular advantage of SVG is the ability to embed scripting commands within the SVG document, which could be used to control the ECG report format (e.g., $3 \times 4,6 \times 2$, $12 \times 1$ row by column waveform layouts, etc.) at the local display client, without a new request to the ECG server.

\section{European OpenECG}

The European Commission sponsored OpenECG project has initiated extensive discussions in the past two years regarding open ECG formats and support for interoperability between medical devices and information systems [9]. The objectives are to identify the driving force and barriers to the diffusion of ECG interoperability standards and to support interoperability efforts in computerized electrocardiography. Philips participated in the meetings and discussions of the OpenECG project, and provided XML-ECG schema and waveform decompression tools to its members [11].

\section{Discussion and conclusions}

The healthcare industry is demanding that electrocardiograph manufacturers open their ECG formats and permit their ECG devices to be interoperable. Interoperability among medical devices and information systems is a cornerstone of the modern healthcare system. The initiatives of standardization organizations and agencies, and the willingness of ECG manufacturers such as Philips to embrace this new direction have already advanced the state-of-the-art of our healthcare delivery system. We welcome other ECG manufacturers to join us in opening their ECG file formats. We are hopeful that the efforts made by Philips Medical Systems and those of the medical device industry as a whole will ultimately lead to a better connected world.

\section{Acknowledgements}

The authors wish to acknowledge their colleagues in the Diagnostic ECG, Patient Monitoring, and Cardiac Resuscitation Divisions of Philips Medical Systems for their concerted efforts in advancing the state-of-the-art of ECG standards and connectivity.

\section{References}

[1] http://www.xml.org

[2] Harold E, Means W. XML in a Nutshell $2^{\text {nd }}$ Edition. O'Reilly \& Assoc. ,Sebastopol, CA. 2002.

[3] Zhou S, et al. New Approaches in Philips ECG Database Management System Design. Computers in Cardiology 2003.

[4] Watt A, et al. SVG Unleashed. Sam's Publishing, Indianapolis, IN. 2002.

[5] http://www.adobe.com/svg

[6] The Clinical Evaluation of QT Interval Prolongation and Proarrhythmic Potential for Non-antiarrhythmic Drugs, FDA Preliminary Concept Paper.

[7] http://www.HL7.org

[8] http://www.himss.org

[9] http://www.openecg.net

[10] http://www.acc.org/quality/ihe.htm

[11] Zhou S, Helfenbein E. OpenECG Format, Philips Experience. OpenECG Workshop Proceeding, 2004.

Address for correspondence:

Eric Helfenbein

Senior Research Scientist

Philips Medical Systems

540 Alder Dr. Bldg 4

Milpitas, CA 95035

Eric.Helfenbein@Philips.com 